Published in final edited form as:

Immunol Res. 2013 December ; 57(0): 81-85. doi:10.1007/s12026-013-8460-5.

\title{
Semaphorin7A: branching beyond axonal guidance and into immunity
}

\author{
Ramon Garcia-Areas, Stephania Libreros, and Vijaya Iragavarapu-Charyulu \\ Department of Biomedical Sciences, Charles E. Schmidt College of Medicine, Florida Atlantic \\ University, 777 Glades Road, Boca Raton, FL 33431-0991, USA
}

Vijaya Iragavarapu-Charyulu: iragavar@fau.edu

\begin{abstract}
Semaphorins are a family of proteins that were originally described for their role in axonal guidance. Studies now show that semaphorins encompass many physiological functions outside of the nervous system, including immune responses. Semaphorin7A (SEMA7A) belongs to the "immune" semaphorin group and has been shown to play a crucial role in regulating immune responses. In this review, we discuss the structure and function of SEMA7A as well as its role of SEMA7A in innate and adaptive immunity. We further describe SEMA7A's involvement in inflammatory disease and its emergent role in cancer.
\end{abstract}

\section{Keywords}

Semaphorin7A; SEMA7A; Axonal guidance; a1 $\beta 1$-Integrin; Inflammation

\section{Semaphorin7A}

Semaphorins are a protein family that plays important roles in many morphological and physiological processes from viruses to humans [1]. Semaphorins are classified based on their structure and protein domains. The structure of the semaphorins consists of a conserved cysteine-rich ( $\sim 500$ amino acids) residue called the "sema" domain, a plexin-semaphorinintegrin (PSI) domain, and variable protein domains [2]. Variations in C-terminal motifs are the key differentiating factors among semaphorins. There are eight classes of semaphorins: Class 1 and 2 are invertebrates, class 3 through 7 are found in vertebrates, and class 8 are viral-encoded [3]. Semaphorins in class 1 and class 4-7 are mainly membrane-associated, whereas semaphorins in class 2,3 , and 8 are mostly secreted [1]. The membrane association/ secretion behavior allows semaphorins to mediate direct cell-cell interactions or chemoattraction/chemorepulsion. Semaphorins were initially described for their role in axon guidance during neurogenesis. It has now been found that semaphorins branch beyond the nervous system to cover an ample overlap of molecular repertoires. Semaphorins are also involved in physiological processes such as organogenesis, immune cell regulation, and vascular growth [2].

Semaphorin7A (SEMA7A) is the only class 7 semaphorin, and to date, it is the only semaphorin that is GPI-anchored [4]. SEMA7A was first identified as CDw108 on erythrocytes and includes the John Milton Hagen antigen. It is located on chromosome 15

(C) Springer Science+Business Media New York 2013

Correspondence to: Vijaya Iragavarapu-Charyulu, iragavar@fau .edu.

Conflict of interest The authors declare that they have no conflict of interest. 
(15q23-24) [5]. It was not until 1998 that CDw108 was found to be part of the semaphorin family, as SemaK-1 [4]. In 1999, it was renamed SEMA7A and made into a separate class of semaphorins based on its unique GPI anchorage [3]. Yamada et al. [5] were the first to clone the human CDw108 cDNA sequence, which encodes for 666 amino acids. The murine counterpart is composed of 664 amino acids and shares roughly $90 \%$ homology with human SEMA7A [4]. Both human and mouse SEMA7A contain a seven-bladed $\beta$-propeller semaphorin $\mathrm{N}$ terminus domain, a plexin, semaphorin, and integrin domain (PSI), an immunoglobulin-like domain, and the characteristic GPI anchor in their C-terminus [6]. It was shown that SEMA7A can dimerize through the Sema and immunoglobulin domains [6].

\section{Semaphorin7A and its receptors}

Semaphorin7A was originally identified as the vertebrate homolog of virally encoded semaphorins A39R and AHV [7]. Like the viral semaphorins, SEMA7A binds a virusencoded semaphorin protein receptor (VESPR) that is also known as plexin $\mathrm{C} 1$. This receptor is expressed by dendritic cells, monocytes, and neutrophils [8]. Unlike the A39R semaphorin, the induction of SEMA7A on monocyte chemotaxis and activation was not found to be mediated through the receptor plexin C1 [9]. Pasterkamp et al. [10] were the first to propose that SEMA7A's RGD motif (Arg267-Gly268-Asp269) can bind to the very late antigen 1 dimer, a1 $\beta 1$, and promote axon growth. Similarly, Suzuki et al. [11] showed that the modulatory effects of SEMA7A on T cell inflammatory responses are also mediated through the very late antigen 1 dimer, a $1 \beta 1$-integrin. In contrast, the binding of endothelial SEMA7A to macrophage av $\beta 5$ integrin in a colitis model induced the production of antiinflammatory IL-10 [12]. These findings depart from the traditional notion that semaphorins signal through plexins and neuropilins. A recent report by Liu et al. [6] revisited the SEMA7A-plexin C1 interaction and showed that the $\beta$-propeller domains of SEMA7A and plexin $\mathrm{C} 1$ can bind in an edge-to-edge manner. Their crystallographic protein findings propose that SEMA7A's RGD is hidden within the protein structure, therefore making it difficult to bind integrins. Although most SEMA7A studies have investigated its role as a ligand, a novel role for SEMA7A as a receptor for $P$. falciparum merozoite-specific TRAP homolog (MTRAP) has been proposed.

In platelets, it has been found that the $80-\mathrm{kDa}$ membrane-bound SEMA7A can be cleaved off the membrane by ADAM-17 [13]. The extracellular soluble version of SEMA7A has been found to have chemotactic properties in monocytes [9] and promote axon branching [10] and melanocyte dendricity [14], among others. In vitro treatment of recombinant SEMA7A with caspase-9 resulted in 24-kDa fragments [15]. They further demonstrate that mutating the conserved VHQDQAYDD motif in SEMA7A impairs caspase-9 cleavage. In vivo, they showed that Apaf-1 is required for caspase-9 cleavage of SEMA7A [15]. However, it is still largely unknown whether the different cleaved forms of SEMA7A serve differential functions in comparison with full-length SEMA7A, and whether the cleaved forms may lead to alternative signaling programs. Future studies will have to elucidate whether binding of secreted or membrane-bound SEMA7A in cis or trans associations can mediate differential effects through integrins, plexins, or other potential co-receptors.

\section{Role of semaphorin7A in innate immunity}

Holmes et al. [9] first showed a functional role for SEMA7A as an activator of human monocytes in vitro. In a dose-dependent manner, soluble SEMA7A was able to induce the production of proinflammatory cytokines: IL-8, TNF-a, IL-6, and IL-1 [9]. To a lesser extent, SEMA7A was also able to induce the production of IL-8, TNF-a, IL-6, and IL-1 in human neutrophils. In both monocytes and neutrophils, IL-8 was most significantly upregulated by SEMA7A. SEMA7A was able to induce a phenotypic change in these human 
monocytes, and skewed them to become $\mathrm{CD} 11 \mathrm{~b}^{+} / \mathrm{CD} 14^{-} / \mathrm{CD} 1 \mathrm{a}^{-} / \mathrm{CD} 83^{+} / \mathrm{CD} 40^{+} / \mathrm{CD} 86^{+}$ dendritic-like cells [9], consistent with the reports of SEMA7A promoting cell dendricity $[10,14]$. Furthermore, it was found that SEMA7A could chemoattract monocytes at femtomolar concentrations but was less effective in promoting neutrophil migration [9]. Recently, hypoxia-elicited endothelial SEMA7A was found to increase neutrophil transmigration across vasculature [16]. SEMA7A-deficient mice showed decreased neutrophil vascular transmigration despite hypoxic stimuli [16]. TGF- $\beta$ also plays a role in skin inflammation as this protein was found to induce increased expression of SEMA7A on human keratinocytes [17]. Increased SEMA7A contributed to skin inflammation by activating monocytes via $\beta 1$-integrin to produce IL-8, while blocking $\beta$-integrin neutralized the effect of SEMA7A [17]. SEMA7A was also found to influence inflammatory processes in a cornea inflammation model by mediating macrophage infiltration to the cornea [18].

Semaphorin7A (SEMA7A) has been shown to exert either a pro- or anti-inflammatory effect depending on the cell type and receptor subunit engaged by this protein $[11,12]$. Contrary to the pro-inflammatory effects associated with SEMA7A, intestinal epithelial cell (IEC)derived SEMA7A was found to negatively regulate development of colitis [19]. Intestinal macrophages maintain quiescent immune response under constant onslaught by commensal bacteria. SEMA7A was found to play a role in the maintenance of quiescence by inducing the production of immunoregulatory cytokine, IL-10, by intestinal macrophages [19]. Furthermore, these same authors showed that SEMA7A-deficient mice exhibit severe signs of dextran sodium sulfate-induced colitis due to reduced levels of intestinal IL-10. These studies in total indicate differential roles of SEMA7A depending on the receptor engagement and cellular sources. SEMA7A is pro-inflammatory through T cell-monocyte interactions via a1 $\beta 1$-receptor [12] and anti-inflammatory through av $\beta 1$-integrin expressed on intestinal macrophages [12].

Macrophages and monocytes not only respond to SEMA7A, but can also express SEMA7A. THP-1 cells treated with respiratory sensitization chemicals showed increased expression of SEMA7A [20]. Human primary monocytes from West Nile virus expressed significantly higher mRNA levels of SEMA7A, compared to normal healthy controls. SEMA7A is also expressed in macrophages from the lungs of idiopathic pulmonary disease patients [21].

\section{Role of semaphorin7A in adaptive immunity}

SEMA7A is expressed in both myeloid and lymphoid lineages [9]. SEMA7A has been reported to be expressed on activated lymphocytes [22]. While this protein is highly expressed on activated T lymphocytes, it is expressed at lower levels in B lymphocytes [23].

Investigation of SEMA7A expression in tissue during immune system development revealed strong signals in thymus tissue with weak expression in the spleen and lymph nodes. Flow cytometric analysis of thymocytes during development showed that $15 \%$ of thymocytes express this molecule. Further analysis of thymic subpopulations revealed expression in double-positive subsets and in $\mathrm{CD} 4{ }^{\text {bright }} \mathrm{CD} 8^{\text {dull }}$ subpopulations with the absence of expression on single-positive CD4 or CD8 T lymphocytes [22]. There is no further expression of SEMA7A in T cell precursors until single-positive $\mathrm{T}$ cells exit the thymus and are activated in the periphery [5]. SEMA7A is expressed in early lymphocyte activation and has been reported to be expressed on most $\mathrm{T}$ and NK cells and B cells as early as $4 \mathrm{~h}$ after activation with maximal expression at $48 \mathrm{~h}$ post-stimuli and then gradually disappears [24]. Thus, this may indicate a specific function that is related to the activation and differentiation of lymphocytes.

In the periphery, SEMA7A has been found to play an important role in the effector phase of cell-mediated immune responses and inducing the production of pro-inflammatory cytokines 
by macrophages. Activated $\mathrm{CD} 4^{+} \mathrm{T}$ cell-expressed SEMA7A has been found to accumulate at the contact zone between T cells and macrophages. Thus, SEMA7A is recruited to lipid rafts in the immunological synapse between $\mathrm{T}$ lymphocytes and macrophages, resulting in clustering of a1 $\beta 1$-integrins and formation of focal adhesion complexes in monocytes and macrophages [11]. Thus, stimulation of macrophages through a $1 \beta 1$-integrins induces the activation of the downstream mitogen-activated protein kinase pathway, resulting in the production of proinflammatory cytokines. While SEMA7A has an activating effect on monocytes, it has been reported to negatively regulate $\mathrm{T}$ cell-mediated immune responses [25]. It was shown that SEMA7A ${ }^{-/-} \mathrm{T}$ cells were hyper-proliferative upon antigenic stimulation. Further, SEMA7A ${ }^{-1-}$ mice had more severe responses in experimental autoimmune encephalomyelitis (EAE) conditions compared to wild-type mice. In contrast to these findings, Okuno et al. [26] reported that SEMA7A-deficient mice immunized with a myelin oligodendrocyte (MOG) peptide in CFA generate MOG-peptide-specific CD4 ${ }^{+} \mathrm{T}$ cells and are resistant to EAE development. Further, it was shown that SEMA7A on antigenprimed $\mathrm{T}$ cells induces inflammation in EAE through interactions with a1 1 1-integrin [11] and that antigen-specific $\mathrm{T}$ cells and cytokine-producing effector $\mathrm{T}$ cells are not impaired in SEMA $7^{-1-}$ mice [11]. These findings indicate that SEMA7A is pathologically involved in EAE.

Regulatory T lymphocytes are important in maintaining homeostasis of the immune system in healthy and pathological conditions. Idiopathic pulmonary fibrosis is characterized by epithelial injury and inflammation with an abnormal wound-healing response [27]. Lymphocytes are reported to be involved in the immunopathogenesis of fibrosis [28-31]. Since abnormalities in regulatory $\mathrm{T}$ cells are seen in lungs of patients with fibrosis, Reilkoff et al. [21] assessed SEMA7A expression in human and mouse models of fibrosis. Increased levels of SEMA7A ${ }^{+} \mathrm{CD} 4{ }^{+} \mathrm{CD} 25^{+} \mathrm{FoxP} 3^{+}$Tregs were observed in the blood of patients with rapidly progressive IPF. It was further shown that SEMA7A-expressing Tregs in contrast to SEMA $7^{-} \mathrm{CD} 4^{+} \mathrm{CD} 25^{+} \mathrm{FoxP} 3^{+}$cells show reduced expression of regulatory mediators, such as IL-10 [21]. It was postulated that since SEMA7A- Tregs were not associated with progressive IPF, SEMA7A expression identifies a population of Tregs that traffic to the lung and contribute to disease progression via impaired suppressor capabilities [21]. To date, the expression of SEMA7A by B cells and the effect of SEMA7A on B cells have not yet been extensively explored. Holmes et al. [23] noted that exogenous SEMA7A did not induce B cell production of proinflammatory cytokines. $\mathrm{CD} 19^{+} \mathrm{B}$ cells from patients suffering from scleroderma-related interstitial lung disease showed high levels of SEMA7A compared to healthy controls.

\section{Semaphorin7A's role in inflammatory diseases}

Inflammation induced by the interaction between SEMA7A and its receptors contributes to inflammation by stimulation of macrophage chemotaxis [9], regulation of monocyte differentiation into dendritic cell type [9], chemokine production, and modulation of T cell function [11]. In its pro-inflammatory role, SEMA7A has been found to play a role in liver fibrosis [32], neuronal injury [33], pulmonary fibrosis [23], skin inflammation [17], corneal inflammation [18], and multiple sclerosis [26]. Murray et al. [34] demonstrated that M2-type macrophages promote transforming growth factor (TGF- $\beta 1$ )-induced lung fibrosis and that SEMA7A regulates TGF- $\beta 1$-induced M2 and fibrocyte differentiation. Additionally, they demonstrated that SEMA7A and its receptors are induced by TGF- $\beta 1$ and that SEMA7A plays a vital role in a PI3 K/PKB/AKT-dependent pathway that contributes to TGF- $\beta 1$ induced fibrosis and remodeling. Gan et al. [23] determined the role of SEMA7A in TGF$\beta 1$-induced pulmonary fibrosis. Using TGF- $\beta$ transgene-positive mice, it was found that SEMA7A mediates fibrocyte accumulation in the TGF- $\beta 1$-exposed lungs by $\beta 1$-integrindependent pathway [23]. In determining the signaling pathway by which SEMA7A induces 
fibrosis, it was observed that TGF- $\beta$ activates PIP-3 K and its downstream Akt pathway in a SEMA7A dependent way [2, 12]. In hepatic fibrosis, the expression of SEMA7A and its receptor $\beta 1$-integrin subunit was up-regulated in hepatic stellate cells (HSCs) during liver injury. Overexpression of SEMA7A in HSCs revealed increased fibrosis with inflammatory mediator expression while SEMA7A gene silenced HSCs has decreased expression of inflammatory mediators [32].

A link between inflammation and hypoxia has been recently noted. In hypoxia-induced inflammation, proinflammatory cytokines are released by the immune cells. SEMA7A was also found to induce the production of cytokines in macrophages and monocytes that play a role during effector phase of inflammatory immune response $[9,11]$. The role of SEMA7A in the development of hypoxia-elicited inflammatory response was determined by MoroteGarcia et al. [16]. This study showed that SEMA7A is significantly induced by hypoxia in a process regulated by the hypoxia-inducible factor. These studies suggest that SEMA7A plays a vital role in varied inflammatory diseases.

\section{Semaphorin7A and cancer}

The involvement of SEMA7A in cancer is still largely unknown. To date, it has been found that SEMA7A is up-regulated in glioblastoma cell line [35]. A recent study has shown a novel role for SEMA7A in regulating epithelial to mesenchymal transition (EMT) in a mammary epithelial cell line. Inhibition of SEMA7A in EpRas cells resulted in an impaired ability to undergo TGF- $\beta$-induced EMT. Our laboratory has found that murine mammary carcinomas, E0771 and DA-3, have increased SEMA7A mRNA levels compared to the expression of nontumorigenic EpH4 mammary cells (Fig. 1a). To date, no published studies have investigated the role of SEMA7A in breast cancer. Similar to its role in migratory function on monocytes, SEMA7A was found to directly induce migration of DA-3 tumor cells in a dose-dependent manner (Fig. 1b). We are currently evaluating the contributions of tumor-derived SEMA7A and its plausible effects on immune cells during breast cancer progression.

\section{Conclusions}

In this review, we have summarized the findings on SEMA7A, the only member in semaphorin family that is GPI-anchored. An immune function for SEMA7A is described as it plays a role in both innate and adaptive immune systems. In the innate immunity, it is a potent stimulator of monocytes inducing the production of pro-inflammatory cytokines. On the other hand, SEMA7A has been shown to function as a regulator of $\mathrm{T}$ cell immune response by its effects on $\mathrm{T}$ cell proliferation. We also describe a novel role for SEMA7A in cancer as it had a direct chemoattractant activity on breast cancer cells.

\section{Acknowledgments}

This work was supported by different National Institutes of Health grants NIH R15 CA135513-01 and R15 CA135513-01-OS1.

\section{References}

1. Capparuccia L, Tamagnone L. Semaphorin signaling in cancer cells and in cells of the tumor microenvironment-two sides of a coin. J Cell Sci. 2009; 122(Pt 11):1723-36. [PubMed: 19461072]

2. Zhou Y, Gunput RA, Pasterkamp RJ. Semaphorin signaling: progress made and promises ahead. Trends Biochem Sci. 2008; 33(4):161-70. [PubMed: 18374575] 
3. Semaphorin Nomenclature Committee. Unified nomenclature for the semaphorins/collapsins. Cell. 1999; 97(5):551-2. [PubMed: 10367884]

4. $\mathrm{Xu} \mathrm{X}$, et al. Human semaphorin $\mathrm{K} 1$ is glycosylphosphatidylinositol-linked and defines a new subfamily of viral-related semaphorins. J Biol Chem. 1998; 273(35):22428-34. [PubMed: 9712866]

5. Yamada A, et al. Molecular cloning of a glycosylphosphatidylinositol-anchored molecule CDw108. J Immunol. 1999; 162(7):4094-100. [PubMed: 10201933]

6. Liu H, et al. Structural basis of semaphorin-plexin recognition and viral mimicry from Sema7A and A39R complexes with PlexinC1. Cell. 2010; 142(5):749-61. [PubMed: 20727575]

7. Pasterkamp, RJ., editor. Advances in experimental medicine and biology. Vol. 600. New York, NY: Springer; 2007. Semaphorins: receptor and intracellular signaling mechanisms; p. XIVp. 147

8. Chabbert-de Ponnat I, et al. Soluble CD100 functions on human monocytes and immature dendritic cells require plexin C1 and plexin B1, respectively. Int Immunol. 2005; 17(4):439-47. [PubMed: 15746246]

9. Holmes S, et al. Sema7A is a potent monocyte stimulator. Scand J Immunol. 2002; 56(3):270-5. [PubMed: 12193228]

10. Pasterkamp RJ, et al. Semaphorin 7A promotes axon outgrowth through integrins and MAPKs. Nature. 2003; 424(6947):398-405. [PubMed: 12879062]

11. Suzuki K, et al. Semaphorin 7A initiates T-cell-mediated inflammatory responses through alpha1beta1 integrin. Nature. 2007; 446(7136):680-4. [PubMed: 17377534]

12. Kang HR, et al. Semaphorin 7A plays a critical role in TGF-beta1-induced pulmonary fibrosis. J Exp Med. 2007; 204(5):1083-93. [PubMed: 17485510]

13. Fong KP, et al. Deciphering the human platelet sheddome. Blood. 2011; 117(1):e15-26. [PubMed: 20962327]

14. Scott GA, McClelland LA, Fricke AF. Semaphorin 7a promotes spreading and dendricity in human melanocytes through beta1-integrins. J Invest Dermatol. 2008; 128(1):151-61. [PubMed: 17671519]

15. Ohsawa $S$, et al. Caspase-9 activation revealed by semaphorin 7A cleavage is independent of apoptosis in the aged olfactory bulb. J Neurosci. 2009; 29(36):11385-92. [PubMed: 19741144]

16. Morote-Garcia JC, et al. Endothelial Semaphorin 7A promotes neutrophil migration during hypoxia. Proc Natl Acad Sci USA. 2012; 109(35):14146-51. [PubMed: 22891341]

17. Kamata M, et al. Semaphorin 7A on keratinocytes induces interleukin-8 production by monocytes. J Dermatol Sci. 2011; 62(3):176-82. [PubMed: 21524887]

18. Namavari A, et al. Semaphorin 7a links nerve regeneration and inflammation in the cornea. Invest Ophthalmol Vis Sci. 2012; 53(8):4575-85. [PubMed: 22700709]

19. Kang S, et al. Intestinal epithelial cell-derived semaphorin 7A negatively regulates development of colitis via alphavbeta1 integrin. J Immunol. 2012; 188(3):1108-16. [PubMed: 22198947]

20. Verstraelen S, et al. Gene profiles of THP-1 macrophages after in vitro exposure to respiratory (non-)sensitizing chemicals: identification of discriminating genetic markers and pathway analysis. Toxicol In Vitro. 2009; 23(6):1151-62. [PubMed: 19527780]

21. Reilkoff RA, et al. Semaphorin $7 \mathrm{a}+$ regulatory $\mathrm{T}$ cells are associated with progressive idiopathic pulmonary fibrosis and are implicated in transforming growth factor-beta1-induced pulmonary fibrosis. Am J Respir Crit Care Med. 2012; 187(2):180-8. [PubMed: 23220917]

22. Mine T, et al. CDw108 expression during T-cell development. Tissue Antigens. 2000; 55(5):42936. [PubMed: 10885563]

23. Gan Y, et al. Role of semaphorin 7a signaling in transforming growth factor beta1-induced lung fibrosis and scleroderma-related interstitial lung disease. Arthr Rheum. 2011; 63(8):2484-94. [PubMed: 21484765]

24. Angelisova P, et al. Characterization of the human leukocyte GPI-anchored glycoprotein CDw108 and its relation to other similar molecules. Immunobiology. 1999; 200(2):234-45. [PubMed: 10416131]

25. Czopik AK, et al. Semaphorin 7A is a negative regulator of T cell responses. Immunity. 2006; 24(5):591-600. [PubMed: 16713976] 
26. Okuno T, Nakatsuji Y, Kumanogoh A. The role of immune semaphorins in multiple sclerosis. FEBS Lett. 2011; 585(23):3829-35. [PubMed: 21420960]

27. Homer RJ, et al. Modern concepts on the role of inflammation in pulmonary fibrosis. Arch Pathol Lab Med. 2011; 135(6):780-8. [PubMed: 21631273]

28. Yoshizaki A, et al. CD19 regulates skin and lung fibrosis via Toll-like receptor signaling in a model of bleomycin-induced scleroderma. Am J Pathol. 2008; 172(6):1650-63. [PubMed: 18467694]

29. Wilson MS, et al. Bleomycin and IL-1beta-mediated pulmonary fibrosis is IL-17A dependent. J Exp Med. 2010; 207(3):535-52. [PubMed: 20176803]

30. Kass DJ, et al. Cytokine-like factor 1 gene expression is enriched in idiopathic pulmonary fibrosis and drives the accumulation of CD4+ T cells in murine lungs: evidence for an antifibrotic role in bleomycin injury. Am J Pathol. 2012; 180(5):1963-78. [PubMed: 22429962]

31. Lo Re S, et al. Platelet-derived growth factor-producing CD4+ Foxp3+ regulatory T lymphocytes promote lung fibrosis. Am J Respir Crit Care Med. 2011; 184(11):1270-81. [PubMed: 21868503]

32. De Minicis S, et al. Semaphorin 7A contributes to TGF-beta-mediated liver fibrogenesis. Am J Pathol. 2013; 183(3):820-30. [PubMed: 23850082]

33. Kopp MA, et al. Spinal cord injury induces differential expression of the profibrotic semaphorin 7A in the developing and mature glial scar. Glia. 2010; 58(14):1748-56. [PubMed: 20645410]

34. Murray LA, et al. TGF-beta driven lung fibrosis is macrophage dependent and blocked by Serum amyloid P. Int J Biochem Cell Biol. 2011; 43(1):154-62. [PubMed: 21044893]

35. Formolo CA, et al. Secretome signature of invasive glioblastoma multiforme. J Proteome Res. 2011; 10(7):3149-59. [PubMed: 21574646] 

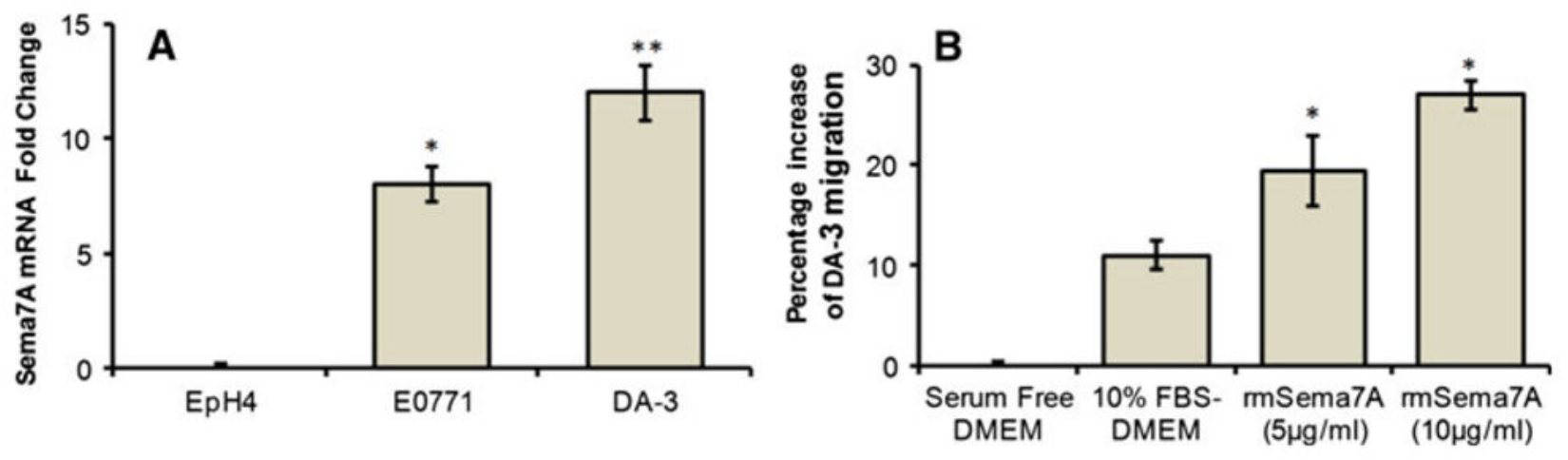

Fig. 1.

SEMA7A gene expression in murine mammary cells. a Total RNA was purified from benign EpH4 mammary cells and E0771 and DA-3 mammary tumor cells and analyzed using qRT-PCR primers (SABiosciences) for the expression of murine semaphorin7A. $\mathbf{b}$ Calcein AM-labeled DA-3 cells $\left(0.1 \times 10^{6}\right)$ were placed in the upper chamber of BD Fluoroblok 0.8- $\mu \mathrm{m}$ transwell inserts in serum-free DMEM with and without rmSEMA7A (R\&D Systems) and incubated for $12 \mathrm{~h}$ using $10 \%$ FBS-DMEM as a positive control. The fluorescent intensity of migrated DA-3 was measured at $12 \mathrm{~h}$ and compared to serum-free DMEM as percentage increase. Data are representative of three independent experiments. Statistical comparisons of paired groups were determined by Student's $t$ tests. $*<0.05, * *$ $<0.01$ 